\title{
Protée
}

\section{Voyage au pays de la peur}

\section{rumeur et récit dans La Classe de neige d'Emmanuel Carrère}

\section{Marie-Pascale Huglo}

Volume 32, numéro 3, hiver 2004

La rumeur

URI : https://id.erudit.org/iderudit/011263ar

DOI : https://doi.org/10.7202/011263ar

Aller au sommaire du numéro

\section{Éditeur(s)}

Département des arts et lettres - Université du Québec à Chicoutimi

ISSN

0300-3523 (imprimé)

1708-2307 (numérique)

Découvrir la revue

Citer cet article

Huglo, M.-P. (2004). Voyage au pays de la peur : rumeur et récit dans La Classe de neige d'Emmanuel Carrère. Protée, 32(3), 101-112.

https://doi.org/10.7202/011263ar

\section{Résumé de l'article}

La Classe de neige d'Emmanuel Carrère entretient avec la rumeur des liens que je me propose d'examiner. Ce récit permet en effet de capter la rumeur à l'état latent, dans l'imaginaire du jeune Nicolas marqué par les contes, les catastrophes des actualités télévisées et les récits donnés pour vrais. De ce tissu narratif traversé par des motifs similaires surgit une histoire qui, dès lors que Nicolas la raconte, actualise la rumeur et son mode de propagation constitutif. En même temps, elle exacerbe les pouvoirs de la rumeur, puisqu'elle provoque le dénouement tragique du récit de Carrère. L'histoire qui se propage ainsi est elle-même une rumeur attestée - celle du trafic des organes - dont j'analyse l'émergence, la transformation, la circulation et la dramatisation dans $L a$ Classe de neige afin d'en ressaisir le régime discursif en lien avec l'intrigue narrative. Ce faisant, ce sont bien les pouvoirs de la rumeur dans la société contemporaine que je vise à souligner.
Ce document est protégé par la loi sur le droit d'auteur. L'utilisation des services d’Érudit (y compris la reproduction) est assujettie à sa politique d'utilisation que vous pouvez consulter en ligne.

https://apropos.erudit.org/fr/usagers/politique-dutilisation/ 


\section{VOYAGE AU PAYS DE LA PEUR: RUMEUR ET RÉCIT DANS LA CLASSE DE NEGE D'EMMANUEL CARRÈre 1}

Dans La Peur en Occident, Jean Delumeau mentionne un test psychologique bien connu des spécialistes de l'enfance: le Test du pays de la peur (Delumeau, 1978: 35). Le séjour du jeune Nicolas en classe de neige que raconte Emmanuel Carrère serait ce test ou, plutôt, cette épreuve. Dès le début du voyage, une angoisse diffuse oppresse le garçon craintif qui trouve, dans les indices les plus infimes, de quoi alimenter sa peur. Sa connaissance du monde est partielle, lacunaire; elle se bute aux non-dits, aux exclusions, aux refoulements des adultes, et reste à l'écart du savoir des autres enfants. L'expérience fragmentaire et limitée de Nicolas est toutefois relayée, médiatisée par les histoires qu'il a lues ou qu'on lui a racontées. Pour lui, le monde est un tissu de fables, un répertoire de schémas narratifs potentiels que la moindre alarme convoque. Les récits qui l'habitent sont, pour la plupart, des histoires épouvantables, elles composent, propagent, identifient et renforcent ce que l'on considère généralement comme négatif: catastrophes naturelles, agressions, accidents, dangers. La Classe de neige ${ }^{2}$ constitue en elle-même une histoire épouvantable: elle raconte comment les fabulations de Nicolas, loin de représenter une évasion imaginaire, révèlent une insupportable réalité qui excède, en horreur, toutes les catastrophes qu'il a pu fantasmer.

Or, cette histoire, que l'on pourrait lire comme un simple drame psychologique, remet en circulation une légende urbaine - celle du trafic d'organe - attestée en Europe, aux États-Unis, en Amérique latine et en Asie ${ }^{3}$. Cela nous invite à examiner le lien qui s'instaure entre la progression narrative de La Classe de neige et la propagation de la rumeur dans le récit. En effet, c'est presque à l'état latent, dans l'univers mental de Nicolas, que Carrère saisit la rumeur, montrant comment elle en vient à «éclore» à partir d'une multitude de motifs qui circulent d'une fable à l'autre. L'intrigue de La Classe de neige se noue à partir du moment où la rumeur du trafic d'organes s'actualise et se transmet, révélant par là même l'intime relation entre la mobilisation imaginaire de la fable et sa circulation effective. En passant du père au fils (Nicolas) et du fils à son redoutable camarade (Hodkann), la fable protéiforme se trouve par ailleurs investie d'une vertu identificatoire et révélatrice qui exacerbe les pouvoirs de la rumeur dans la société contemporaine. 


\section{LE PAYS DE LA PEUR}

Le pays de la peur est une contrée fantomatique où les représentations imaginaires de Nicolas peuplent un réel saturé de virtualités. On le voit dès le chapitre un: l'éventualité d'un accident, qu'évoque son père pour expliquer un bouchon sur les petites routes conduisant au chalet, ne se concrétise pas: Nicolas ne voit rien des «voitures en accordéon» et des «corps sanglants» (p. 11) qu'il anticipe. Entre ses attentes dramatiques, la nullité du spectacle et l'incertitude d'une réalité hypothétique, un «mystère» (p. 11) subsiste. Une zone trouble, latente, située à la croisée de l'imaginaire et de la réalité, prend corps dans la peur et s'évanouit quand elle disparaît. Dans cette première scène, l'image fantasmatique de l'accident est rapidement évoquée, mais on comprend, dès l'ouverture du chapitre deux, qu'elle répond au drame montré dix jours plus tôt aux informations télévisées: "un poids lourd ayant percuté un autobus scolaire, plusieurs enfants étaient morts atrocement brûlés " (p.12). Cette nouvelle déclenchera le réflexe protecteur du père de Nicolas, qui préférera conduire lui-même son fils à la classe de neige plutôt que de le laisser faire le trajet avec les autres enfants en autobus, mais il déclenche aussi l'anticipation du drame dans l'esprit du garçon. L'épisode reste anodin, il passe aussi vite que le bouchon sur la route de montagne et pourtant, il constitue, au même titre que le traitement à part de Nicolas et la mort des enfants accidentés, une amorce. Car ce n'est pas seulement la situation malaisée de Nicolas au sein du groupe de la classe de neige et le meurtre du jeune René qui se profilent làdedans, c'est aussi, et surtout, la multiplication des récits éphémères qui répondent à un monde incertain et aux peurs qu'il suscite. La hantise de la réalité à laquelle l'imaginaire de Nicolas donne un corps virtuel ne cesse de se métamorphoser au gré des indices et des motifs d'inquiétude.

La voix narrative, qui passe de la vision subjective du «héros» à son univers intérieur, mêle dans son discours les récits lus, vus ou entendus, les catastrophes redoutées et les incidents vécus. Entre les histoires que le jeune garçon se remémore et les drames qu'il anticipe, un réseau de correspondances se met en place que la voix actualise et répercute. Ainsi, le tropisme ${ }^{4}$ qui s'épanouit à la faveur d'une blague cruelle de Hodkann, qui fait la loi dans le dortoir, est une amplification dramatique d'une réalité directement en prise avec le monde imaginaire:

"-Moi, dit Hodkann d'une voix paisible, si j'étais ton père, je me servirais de toi pour faire les démonstrations. Je te couperais les bras et les jambes, j'adapterais les prothèses et je te montrerais comme ça à mes clients. Ça ferait une bonne publicité». Les occupants du lit voisin éclatèrent de rire, Lucas dit quelque chose au sujet du capitaine Crochet, dans Peter Pan, et Nicolas eut peur, tout à coup, comme si Hodkann montrait enfin son vrai visage, encore plus dangereux qu'il ne l'avait redouté. Les hommes de main, serviles, commencent déjà à rire tandis que le potentat cherche nonchalamment dans son imagination le plus raffiné des supplices.

(p.29; je souligne)

Le tropisme au présent est une dramatisation intérieure de la situation: la blague se métamorphose en torture, les rires en servilité, le chef en potentat qui, par la cruauté, s'apparente au capitaine Crochet. Le scénario qui, brièvement, s'esquisse, ne nous est pas inconnu, il réactive la mémoire de Peter Pan (le capitaine cruel et ses vils hommes de main) et des récits de torture qui, fictifs ou non, peuplent l'imaginaire collectif. Nicolas est une sorte de corps conducteur qui, de l'extérieur à l'intérieur, donne à voir la médiation conjointe de la mémoire et de l'imaginaire dans notre saisie affective du réel.

Par ailleurs, la voix narrative est porteuse de motifs qui, d'une scène à une autre, se déplacent, se recoupent et forment un tissu dense de correspondances. Entre le capitaine Crochet et les démonstrations publicitaires, le motif de la mutilation se répète; entre les figures du père, du capitaine et de Hodkann, l'ombre du tortionnaire se profile; entre le visage paisible de Hodkann et son "vrai visage», le lieu commun de la vérité cachée sous des apparences trompeuses trouve à s'actualiser ${ }^{5}$. De là, tout un réseau de correspondances se met en place: mutilations des corps des enfants atrocement brûlés 
dans l'accident d'autobus vu aux informations télévisées, morcellement du bonhomme anatomique que Nicolas souhaite gagner avec les bons de la station Shell, morcellement du corps des enfants dont on vole les organes, fils déchiqueté par accident dans les Histoires épouvantables, etc. Sous la réalité inquiétante couvent donc des peurs et des schèmes narratifs très anciens, prêts à éclore au moindre signe.

Le même Hodkann se métamorphosera plus loin en tigre dans l'esprit de Nicolas cherchant à anticiper ce qui arrivera s'il a le malheur de faire pipi au lit (p.31). Dans ce tropisme, on reconnaît l'image du prédateur et de sa proie que nous avons vue poindre, plus haut, avec le vrai visage (cruel) caché sous des apparences trompeuses: le tigre remplace le potentat, révélant ainsi la malléabilité de l'imaginaire dans le cadre bien circonscrit du lieu commun. À cette malléabilité quasi inépuisable se joint l'évidence implicite de l'image: il n'est pas nécessaire d'aller au bout du tropisme, d'en déplier le drame, pour comprendre. Les schèmes imaginaires esquissés sont suffisamment intégrés dans la mémoire collective pour qu'une simple amorce parvienne à en réveiller le potentiel. C'est bien à titre d'événement en puissance que le tropisme trouve sa pleine efficacité, et l'ébauche narrative suffit pour donner à la menace un visage précis sans en épuiser la potentialité génératrice.

Dans le pays de la peur, où la crainte de l'avenir prête un visage changeant, protéiforme, à «l'ennemi», les drames narratifs imaginés par Nicolas lui permettent d'orienter son angoisse en fonction des stimuli extérieurs. Les catastrophes envisagées se résorbent au fur et à mesure que progresse le récit, dans un mouvement inquiet qui relance, sous d'autres formes, les mêmes hantises désignant chaque fois autrement «la chose sans nom» (p.25) qui paralyse l'enfant. Comme le bouchon qui disparaît dans le chapitre un, la "surprenante douceur» (p.29) de Hodkann résorbe l'image du potentat. Les drames sont éphémères, informulés, ils apparaissent, disparaissent et tissent un réseau serré d'incidents qui se relaient et s'amplifient tout en relançant chaque fois, pour Nicolas, l'espoir de s'en sortir.
À la crainte de faire pipi au lit répond ainsi la «catastrophe» (p. 67) de la pollution nocturne. Nicolas ne connaît rien de sa sexualité. Cela ouvre la voie à d'autres histoires, d'autres hantises ${ }^{6}$, qui l'amènent à croire qu'il se liquéfie dans le noir : «c'était pire qu'une plaie, cela suintait de lui. Bientôt ce serait lui» (p.68). D'angoissante, la situation devient insupportable à partir du moment où le danger d'être rejeté vient non plus des autres (du groupe), mais de lui-même, qui se vide: «Il avait peur, peur d'eux, peur de lui-même. Il pensa qu'il fallait s'enfuir, se cacher, se liquéfier seul, loin de tous. C'était fini pour lui. Plus personne ne le reverrait» (p.69). Cette dramatisation entraîne la fugue de Nicolas "au cour de la nuit» (p.69). Il s'agit d'un point tournant dans le récit: l'imaginaire se renverse en réalité et met en danger la vie du jeune garçon. Il passe la nuit dehors, transi de froid, dans la voiture du moniteur, seul refuge dans un monde protéiforme: le chalet endormi ressemble à "un gros animal repu ( p.71), le bruit d'une branche contre la vitre exacerbe la peur «[m]oins d'une bête que d'un assassin rôdant la nuit autour du chalet, prêt à dépecer les enfants [...]»(p.76). Nicolas sera retrouvé et sauvé au petit matin, mais une autre disparition confirmera le basculement du cauchemar dans la réalité que sa fugue amorce. Dans l'économie du récit, cette fugue marque en effet la marche «inexorable» de quelque chose de "terrible, qui ne s'arrêtera pas" ${ }^{7}$ : après Nicolas, c'est René, un garçon du village, qui disparaît. On le retrouvera assassiné, ce qui amènera Nicolas à confier un secret à Hodkann, à verbaliser sa peur. Mais en rompant avec le régime des histoires latentes qui couvent en silence, il signe sans le savoir l'arrêt de mort de son père et, par extension, le sien.

La transmission d'un secret initialement transmis à Nicolas par son père correspond au stade de l'incubation de la rumeur qu'Edgar Morin identifie en ces termes:

[...] l'incubation s'est d'abord effectuée dans les classes de jeunes filles [...], doublement propices à la transmission et à la prolifération fantasmatique, d'une part parce que ces milieux constituent de véritables caisses de résonance et d'amplification, d'autre part parce que leur population 
adolescente [...] vit dans l'inexpérience du monde social.

(Morin, 1969: 23-24)

Ce stade ne sera pas dépassé dans le récit de Carrère: la rumeur du trafic des organes sera tuée dans l'œuf avec la trahison de Hodkann et l'arrestation du père de Nicolas. Le récit ne prend même pas suffisamment d'ampleur pour transformer le milieu de la classe de neige en "caisse de résonance». En ce sens, Carrère coupe la rumeur $\mathrm{du}$ "processus de contamination orale» qui la caractérise (Campion-Vincent, 1988: 75): aucun foisonnement, aucune prolifération, aucune circulation au sein de la micro-société des élèves ou du village n'aura lieu. Quelque chose d'indissociablement lié à la rumeur néanmoins se noue.

\section{LES TANIÈRES DE L'ARKHÈ}

Pour Edgar Morin, la rumeur est un mythe dans lequel les archétypes nourrissent et se nourrissent des éléments de la réalité. L'imaginaire archaïque de la mort, qui prend, dans La Classe de neige, différents visages, est un milieu de fermentation dont les tropismes et les fables remémorées montrent bien la malléabilité. Si la rumeur ne circule pas ou à peine, les fables intérieures, elles, prolifèrent et révèlent, dans leur multiplication, les déplacements d'un même archétype sous différentes formes: le tigre, les bêtes, l'ogre, l'accident, le capitaine Crochet ou le voleur d'organes rejouent un meurtre confusément désiré ou craint. L'inquiétude de ne pas voir son père revenir lui rapporter le sac de voyage (contenant pyjama, alèse, etc.) oublié dans le coffre de sa voiture, comme il aurait normalement dû le faire, provoque l'anticipation imaginaire de sa mort par accident de voiture. Cela métamorphose Nicolas en «orphelin, héros d'une tragédie» (p.45), puis en coupable («Il serait non seulement orphelin, mais coupable, terriblement coupable. Ce serait comme d'avoir tué son père» [p.61]). Parallèlement, la peur d'être tué ne trouve pas à se fixer. Les méchants sont ici les trafiquants d'organes, là le capitaine Crochet, là encore Nicolas lui-même se liquéfiant. La victime est tantôt le petit frère de Nicolas, tantôt Hodkann ou
René, dont l'identité se fond avec la sienne:

Derrière les lunettes, ses yeux se noyaient d'épouvante, l'épouvante d'un petit garçon sur qui un inconnu se penche pour le tuer, et Nicolas sentait se coller sur son propre visage l'expression de René [...]. (p. 106)

La co-présence de contes, de récits entendus, de cauchemars et d'actualité médiatique montre que l'arkhè travaille activement dans le mélange inextricable de bribes véridiques et de fables, d'onirisme et d'information. Un tel mélange constitue, selon Edgar Morin la structure mythique de la rumeur. Le mythe, dans ce cas, «fait communiquer les tanières de l'arkhè, où règnent à l'état élémentaire le désir et l'angoisse, [...] avec la trivialité empirique de l'univers [...] de chacun et de tous" (Morin, 1969: 65). La succession rapide des histoires fait sortir la peur et le désir de leur tanière sans qu'ils réussissent à se fixer, à s'orienter, à se dire. Les récits imaginés et remémorés restent pris dans les signes versatiles d'une réalité éphémère qui se contracte, puis se résorbe, comme un bouchon sur une route de montagne. La communication entre la réalité et la légende reste intermittente, éparpillée dans des signes qui ne font pas corps, dans des récits qui se succèdent sans véritablement stabiliser ensemble le monde contemporain et l'imaginaire archaïque.

L'alternance d'histoires hétérogènes tantôt réalistes, tantôt fabuleuses (les contes), montre que le processus de la circulation et du foisonnement, caractéristique de la rumeur qui "court», participe aussi de son émergence silencieuse. Carrère construit son intrigue à partir d'un ensemble de fables qui situe l'incubation non pas dans la propagation de la rumeur en milieu clos relativement homogène, mais dans une conscience individuelle travaillée par des récits de provenances diverses et des indices variables. Au lieu de définir la rumeur comme un phénomène social, il la saisit dans sa fermentation individuelle pétrie de mémoire collective. Dans cette perspective, le milieu n'est pas le groupe mais l'adolescent, la rumeur n'est pas d'abord conçue comme une histoire relayée par des individus, mais comme un 
papillonnement de fables qui surgissent dans une conscience repliée sur elle-même. La narration romanesque, qui relie ici l'intériorité de Nicolas au monde qui l'entoure (l'effraie), est un dispositif capable de faire apparaître l'émergence de la rumeur avant même sa transmission effective ${ }^{8}$. Elle la saisit dans l'œuf, in vivo, et déploie l'enchevêtrement de l'individuel et du collectif. Là ou les sociologues et les anthropologues saisissent la rumeur à l'intérieur d'un groupe, la rumeur proliférante, le romancier la saisit en phase de latence comme potentialité.

Il est donc intéressant de noter que, par le biais de la médiation romanesque qui fait apparaître la rumeur dans la conscience de Nicolas, on retrouve à l'état élémentaire des traits qui, dans la légende urbaine, se mêlent et se confondent ${ }^{9}$. Dans une rumeur déclarée, la dimension archaïque doit être décryptée sous les lieux réels et les détails plausibles. Edgar Morin relève ainsi dans la rumeur de la traite des blanches les motifs anciens du réseau souterrain de la ville, des catacombes, des puissances occultes. La ville d'Orléans se voit minée, «vidée de l'intérieur» (Morin, 1969: 60) (comme Nicolas la nuit de sa fugue): «C'est la grande ville qui, sous son vernis lumineux et bruyant [...], sous son pouvoir civil, cache un labyrinthe souterrain où règne une puissance occulte» (ibid.: 61). Ces motifs se mêlent aux détails véridiques et sont d'autant plus crédibles qu'ils refoulent, sous le couvert de la plausibilité, des schémas archaïques. Dans La Classe de neige, l'arkhè se révèle dans la force première de son émergence, alors qu'elle ne s'est pas encore fondue tout entière dans la légende urbaine. Elle est le terreau, le terrain élémentaire de la rumeur.

Si l'imaginaire peut se charger d'érotisme (c'est le cas avec l'image «bouleversante» de la petite sirène découvrant ses jambes [p. 74] et la rencontre de "la fée» dans un relais d'autoroute, avec laquelle Nicolas souhaite aller «loin, très loin» [p. 144]), il est le plus souvent rempli d'effroi. Les craintes renouvelées de Nicolas, sa peur sans cesse ressuscitée, soulignent l'enracinement profond du mythe dans l'angoisse, dans un malaise diffus lié au changement, à l'inconnu: la classe de neige sort le garçon de son milieu de vie habituel et le laisse démuni, sans refuge. Entre cette situation pénible, où le danger peut surgir de partout sous n'importe quelle forme, et les «problèmes mal définis» que la rumeur, selon Michel Louis Rouquette, résoud, on peut établir un lien ${ }^{10}$. La "négativité» de la rumeur, sa «noirceur» (Kapferer, 1995: 154) sont ancrées dans une réalité problématique qui fait resurgir nos croyances archaïques dans les forces obscures, souterraines. Et c'est quand l'angoisse atteint le groupe tout entier avec le meurtre de René, quand la nécessité de trouver et de punir un coupable se fait sentir, que le groupe se transforme à son tour en milieu favorable à l'éclosion de la rumeur.

\section{TRANSMISSION ET PERFORMANCE}

Lorsque Nicolas dit son secret à Hodkann, les villageois sont inquiets et les gendarmes recherchent le jeune René: le récit intervient entre la disparition et le meurtre de la victime, qui viendra confirmer la plausibilité du trafic d'organes. Nicolas croit être le premier à annoncer à Hodkann la disparition de René, mais il se trompe: tous les élèves sont déjà au courant. Alors que Hodkann lui demande s'il a une piste, il se lance:

Tout à coup, une idée hui vint, qui l'éblonit. Son père hui avait bien dit de ne jamais en parler, de ne pas trahir la confiance que lui avaient faite les chefs de clinique, mais Nicolas s'en moquait: Hodkann et René valaient cela. (p. 98)

Partant de la nuit de sa fugue (que la maîtresse a prise pour une crise de somnambulisme), il transforme et grossit les indices inquiétants, évacuant toute incertitude. La «lumière jaune qui se déplaçait en contrebas", "sans doute les phares d'une voiture» (p.72), et l'idée d'un «assassin rôdant autour du chalet, prêt à dépecer les enfants qui auraient l'imprudence de s'en écarter» (p.76), disparaissent au profit d'un récit où le doute n'a plus sa place. Nicolas y joue le rôle privilégié du héros-témoin qui en sait plus que les autres:

[...] la nuit où je suis sorti, ce n'était pas une crise de somnambulisme. Je n'arrivais pas à dormir et à un moment, de 
la fenêtre du couloir, j'ai vu de la lumière sur le parking. Un homme se promenait avec une lampe torche. Ça m'a paru bizarre, alors je suis descendu. En me cachant, je l'ai suivi jusqu'à une camionnette blanche, exactement comme celle où ils cachent leurs tables d'opération. L'homme est monté, il a démarré. Les phares étaient éteints, il n'a même pas mis le moteur mais commencé à descendre la route en roue libre, pour ne pas faire de bruit. Ça m'a paru louche, tu comprends. J'ai repensé à cette histoire de trafic d'organes et je me suis dit qu'ils devaient rôder autour du chalet, au cas où quelqu'un sortirait seul... (p.99)

Comme la voix narrative le fait remarquer, «il improvisait, mais déjà toute une histoire prenait corps devant lui, tout ce qui s'était passé les derniers jours trouvait une explication [...]». Autrement dit, ce qui ne parvenait pas à se fixer se cristallise autour de René devenu, par les vertus du récit, victime des trafiquants d'organes. Pour que Hodkann n'évente pas le secret (et ses mensonges), Nicolas invente des policiers complices du trafic et fait de son père un enquêteur solitaire qui, sous prétexte de le conduire à la classe de neige et sous couvert de vendre des prothèses, traque les criminels dans la région, à l'insu de la police et de tous:

Il a un compte à régler avec eux. L'année dernière, ils ont enlevé mon petit frère. Il a disparu dans un parc d'attractions et on l'a retrouvé plus tard derrière une palissade. Ils lui avaient pris un rein. (p. 101)

L'histoire improvisée rassemble les mystères qui, depuis le début de la classe de neige, ont tenu le jeune garçon en alerte - son père qui n'est pas revenu lui ramener son sac de voyage, René qui a disparu - dans un ensemble cohérent qui transforme les indices alarmants en conclusion logique, plausible. La hantise diffuse devient récit véridique. Même Nicolas, «oubliant que tout reposait sur un mensonge de sa part» (p. 100), en vient à croire à sa fabulation. Le danger est ainsi nommé, cerné, mais il est aussi partagé. Il réunit le père, le fils et le grand-frère d'adoption (Hodkann) dans le secret. Le fait de savoir ensemble ce que les autres ignorent les fonde en communauté: «Tu l'a échappé belle si ça se trouve», dit Hodkann à Nicolas (p.99), puis: «On est dans le même bateau» (p. 102). La confidence que Nicolas propage en racontant à Hodkann ce qu'il devrait garder pour lui les "met à part» 11 du reste de la classe et du monde, mais ensemble. Le secret est un objet de partage, un objet relationnel, d'où cette insistance apparemment paradoxale à interdire qu'on le répète au moment même où on le répète: "[...] il ne faut pas que tu en parles, ni à tes copains ni à personne», dit le père de Nicolas quand il lui explique le trafic d'organes (p.33); «Il ne faut le dire à personne [...]» (p.99) demande Nicolas à Hodkann. À ce rituel s'en ajoute un autre. Pour Nicolas comme pour son père, la rumeur ne relève pas d'un «on dit», mais provient d'une source bien informée qui garantit l'authenticité du secret ${ }^{12}$. Bien qu'elle reste inaccessible, cette source inscrit le récit dans une filiation presque directe qui distingue, là encore, ceux qui partagent le savoir du reste de la collectivité (coupable ici, innocente là). Ce qui se transmet par le récit de la rumeur, ce qui se propage, c'est bien la communauté du secret.

Cette communauté en expansion représente un pouvoir illicite, officieux, qui contrebalance les forces officielles corrompues en qui l'on ne peut plus faire confiance. Elle constitue, dans les termes de Kapferer, un contre-pouvoir (1995: 26), et exprime, selon Françoise Reumaux, «une désentrave des liens et une forme d'affranchissement social» (1988: 55) capable de soulever les foules. La rumeur du trafic d'organes n'ira pas jusqu'à provoquer l'émeute, mais le climat est bel et bien explosif:

Dès que l'autocar s'arrêta sur la place du village, [...] Nicolas comprit qu'il était arrivé quelque chose de grave. Un groupe d'une dizaine de personnes [...] se tenait devant le café [...].

L'autocar en se garant attira des regards hostiles. (p. 104)

Il est clair qu'à ce moment-là, tout étranger devient suspect, voire coupable. Comme Nicolas catapulté contre son gré en classe de neige, la collectivité des élèves et des villageois traverse une situation de crise ${ }^{13}$.

Nicolas rassemble et redistribue dans son récit les indices et les mystères cumulés pendant son séjour en 
classe de neige, mais il intègre des éléments du trafic d'organes que son père lui a racontés, qu'il transforme et déplace. Les motifs de l'enlèvement, de la palissade, de la camionnette, des méchants qui rôdent sont repris, de même que le personnage du petit frère impliqué dans le récit d'origine transmis à Nicolas par son père:

C'est une chose que j'ai apprise d'un directeur de clinique, les médecins sont tous au courant mais on ne veut pas que ça se sache, pour ne pas affoler les gens. Il n'y a pas longtemps, dans un parc d'attractions comme celui-ci, un petit garçon a disparu. [...] On l'a cherché toute la journée et le soir on a fini par le retrouver, sans connaissance derrière une palissade. On l'a emmené à l'hôpital, on a vu qu'il avait un gros pansement dans le dos, avec du sang qui coulait, et alors les médecins ont compris [...]: on avait opéré le petit garçon, on lui avait enlevé un rein. Il y a des gens qui font ça figure-toi. Des gens méchants. Ils ont des camionnettes avec tout le matériel pour opérer, ils rôdent autour des parcs d'attractions, ou près de la sortie des écoles, et ils enlèvent des enfants. [...] Tu comprends, maintenant, pourquoi je ne voulais pas confier ton petit frère à un inconnu? (p.34)

De la même façon que le récit se propage d'une personne à l'autre, les motifs de la rumeur se propagent et se transforment d'un relais à l'autre, ils s'amalgament avec d'autres motifs tirés de la réalité immédiate pour former un récit qui se tient: «Cela se tenait terriblement» (p. 100). Le récit circonscrit et nomme "la chose sans nom» sans la figer. Le noyau de la rumeur variable tient dans la légende qui trouve, dans ses actualisations éphémères et changeantes, de quoi satisfaire la croyance. Le lieu commun du vrai visage de la réalité sous des apparences trompeuses coïncide avec la réalité empirique du moment, la figure de l'ogre se confond avec celle des hommes méchants, le trafic d'organes identifie un danger jusque là diffus et explique les mystères restés jusque là inexpliqués. En somme, c'est au moment de la transmission du secret que le récit performé dans l'instant devient rumeur active, actualisée: il fonde une communauté complice, cerne ce qui papillonnait, conjoint imaginaire et réalité dans un schéma plausible, canalise des peurs anciennes et présentes qui trouvent leur explication et, du même coup, leur profonde vérité: celle du mythe. La transmission est une fabulation identificatoire dont on peut suivre, dans le roman de Carrère, les déplacements microscopiques.

\section{RÉCIT ET RÉSEAUX}

Si, dans La Classe de neige, la rumeur ne se propage pas, elle reprend tacitement un motif d'une légende urbaine attestée dans les années 1990: «Le rein volé» (Campion-Vincent et Renard, 1992: 337-344). Le récit transmis par Nicolas à Hodkann s'intègre dans un discours social plus large qui l'identifie comme une rumeur active. Les victimes du trafic sont variables: des touristes européens en voyage au Brésil ou en Turquie, des célibataires séduits dans un bar, des joggers happés dans Central Park à New York ou de simples patients. Dans une des variantes, ce sont les enfants pauvres qui font l'objet du trafic:

La mafia des organes écume les bidonvilles.

$\grave{A}$ onze ans, Oscar s'est fait enlever dans une rue malodorante de la cité misérable où il vit dans la banlieue sud du grand Buenos Aires. On l'a interné dans une clinique qu'il est incapable d'identifier. On lui a enlevé un rein et on l'a ramené à la maison un mois plus tard, avec 400 dollars en poche et une cicatrice.

Oscar est une victime des trafiquants d'organes qui terrorisent les bidonvilles et quartiers pauvres d'Argentine à la recherche de reins qu'ils revendront sur le marché parallèle quelque 45000 dollars à des patients nord-américains, brésiliens ou argentins, qui attendent désespérément une greffe.

Cela fait deux ans que l'on parle en Argentine d'enlèvement d'enfants retrouvés avec une cicatrice et un rein en moins. Mais il s'agit peut-être d'une fantasmagorie collective, d'une gigantesque rumeur qui parcourt d'ailleurs la plupart des pays d'Amérique du Sud.

(M. L. Avignolo, Libération, 12 décembre 1991. Ibid.: 341)

Dans cette version, la rumeur s'amorce comme un récit véridique que la journaliste dramatise avec force pathos et raconte comme une vérité: elle personnalise la victime ( Oscar»), donne des détails réalistes ( 400 dollars en poche») et fait du garçon de onze ans le témoin direct de sa mésaventure («une clinique qu’il 
est incapable d'identifier»). Loin d'affaiblir la crédibilité de l'information, les impasses du témoignage l'authentifient tout en la rendant invérifiable. De là, la journaliste infère une généralité, pour ensuite mettre en doute la véracité du trafic des organes: ce serait un bruit diffus, un «on-dit». En somme, elle authentifie et démystifie la fable, la démystification (généralisante) contribuant à authentifier l'information. Nous pouvons voir s'esquisser, dans ce mouvement argumentatif, l'ambiguité des grands médias vis-à-vis de la rumeur.

Véronique Campion-Vincent et Jean-Bruno Renard associent cette rumeur à une tension sociale issue, d'une part, des inquiétudes reliées au progrès de la technologie médicale, d'autre part, au marché des organes creusant le fossé entre riches et pauvres. Ces paramètres généraux mettent en évidence les cristallisations collectives de la rumeur (que l'écriture contribue à fixer), mais ils ne permettent pas de suivre le processus de la transmission. Les compilations ne font pas apparaître le récit en acte dans sa circulation orale circonstanciée, actualisée d'un « relais» à l'autre. En outre, le souci du contemporain coupe la légende urbaine répertoriée aujourd'hui d'un fonds collectif plus ancien. La rumeur compilée par les folkloristes et les journalistes couvre certes toute la superficie du globe, mais elle semble détachée d'un imaginaire collectif qui n'a pas attendu l'ère des nouvelles technologies pour surgir. Jean Delumeau raconte ainsi des rumeurs relativement anciennes dont le noyau ressemble étrangement au trafic des années 1990:

En 1768, le collège des oratoriens de Lyon est envahi par la foule et saccagé. On accuse les religieux d'héberger un prince manchot. Tous les soirs, raconte-ton, on arrête autour du collège des enfants auxquels on coupe un bras pour l'essayer au prétendu prince. L'émeute fait 25 blessés.

(Delumeau, 2003: 226)

Une rumeur similaire aurait provoqué un bain de sang à Paris puis à Toulouse en 1750 , renaissant chaque fois sous une forme différente. Dans tous ces récits, l'imaginaire du corps morcelé recoupe celui du sacrifice de l'innocent, dont le motif archaïque court d'une rumeur à l'autre. Sacrifice des enfants du peuple pour le prince d'un côté, sacrifice des pauvres pour le bénéfice des riches de l'autre. Dans les deux cas, le terrain est «tendu », conflictuel: «au-delà d'un certain seuil», quand «le signe équivoque devient parfaitement clair» (Morin, 1969: 41), il faut trouver un bouc émissaire (dont l'identité varie selon les circonstances) et le sacrifier à son tour.

Ces quelques rapprochements contribuent à établir le réseau interdiscursif 14 très étendu que sous-tend la rumeur du trafic d'organes dans La Classe de neige. Ils montrent aussi que la circulation des motifs caractéristiques de la légende travaille le roman de l'intérieur. Car si la rumeur ne se propage ni dans le groupe des élèves ni dans celui des villageois, les motifs du rapt d'enfants et du vol d'organes se déplacent de la version du père à celle du fils pour constituer le noyau identificatoire du mythe. Ces motifs eux-mêmes condensent un ensemble de fables et d'incidents réels d'où émerge la rumeur livrée comme une histoire véridique. En somme, la fabrique instantanée du récit chronologique et réaliste que Nicolas livre à Hodkann renvoie à une constellation partiellement légendaire, à un réseau de motifs et de détails qui forment le tissu mouvant de la rumeur en circulation. Or, cet enchaînement logique qui, d'un mélange de traits dispersés tire une histoire circonstanciée, opère aussi au niveau de l'intrigue de La Classe de neige, comme si la rumeur gouvernait le roman dans son entier.

De même que les tropismes et les micro-fictions tracent un parcours en arabesque suivant une logique associative discontinue pour se cristalliser ensuite dans le récit réaliste et linéaire de la traque des trafiquants d'organes autour du chalet, de même les incidents épars que l'on croyait aléatoires finissent par se nouer dans une logique romanesque de cause à effet: entre l'accident de l'autobus scolaire vu à la télévision, la décision du père de Nicolas de conduire son fils luimême au chalet, l'oubli du sac de voyage dans le coffre de la voiture, la catastrophe nocturne, la fugue de Nicolas, la disparition de René, le secret transmis à Hodkann, le meurtre de René et l'arrestation de son père, tout «se tient» terriblement. Le destin se joue de 
hasards, sa dramatisation est d'autant plus efficace que ce qui semblait nous éloigner de la catastrophe nous y ramène: les imprévus du voyage et de la rumeur entraînent ce qui devait arriver depuis toujours.

\section{POUVOIRS DE LA RUMEUR}

La rumeur octroie à celui qui la transmet un pouvoir indubitable. Elle permet au père de contrôler son fils en lui communiquant la peur des étrangers, de maintenir son emprise sur lui. Comme le fait remarquer Jean Delumeau à propos des rumeurs de rapt d'enfants, ces histoires font écho «à la conviction jadis largement répandue que des criminels volaient les enfants» et autres récits de croquemitaines destinés aux marmots désobéissants (Delumeau, 2003: 227). Si les croquemitaines ont aujourd'hui disparu, les maniaques urbains (rôdeurs à l'anesthésique, piqueurs aux seringues contaminées par le sida, pousseurs de métro, balafreurs, etc.) sont bel et bien vivaces dans la mentalité collective (voir à ce propos CampionVincent et Renard, 1992). La peur que le récit des voleurs d'organes distille est, pour le père, un instrument de pouvoir et de contrôle très efficace: la rumeur plonge Nicolas dans la terreur, il fait des cauchemars à répétition et voit le danger partout. En même temps, la rumeur nimbe celui qui la rapporte d'une aura: le père est dans la confidence des chefs de clinique, il sait des choses que "les gens» ignorent. Le prestige lié à un savoir privilégié rejaillit sur celui qui le transmet et projette de lui-même une image favorable: le père n'est plus, à ce moment-là, cet homme tendu qui souffre «de fatigue et d'horaires mal fichus [...]»(p.35). De la même façon, Nicolas devient, dans le récit qu'il fait à Hodkann, un enquêteur téméraire et un témoin privilégié digne d'un héros du Club des Cinq, auquel il s'identifie (p.98). La rumeur transforme le petit dont on se moque en initié que l'on écoute: «Nicolas le toisa. Cette nuit, c'était Hodkann qui posait les questions de timides bon sens, et lui, Nicolas, qui le clouait avec des réponses sibyllines» (p. 100). Dans les deux cas, la rumeur fait du secret partagé un instrument de prestige social. Le père veut dominer et contrôler son fils, Nicolas veut se rapprocher du redoutable Hodkann, en faire son complice.

Seulement voilà. Si la rumeur procure un pouvoir, elle est elle-même une force dangereuse que l'on doit contrôler. Il ne faut pas parler pour ne pas affoler les gens, commande le père à son fils, il faut jurer de garder le silence pour ne pas mettre son père en danger, demande Nicolas à son complice. Alors même que Hodkann jure de se taire, Nicolas anticipe les conséquences désastreuses de son récit s'il venait à s'ébruiter:

Hodkann jura. Nicolas jouissait de l'empire que son récit prenait sur lui. [...] lui aussi maintenant avait un père aventurier, un justicier courant mille dangers, engagé dans une histoire dont il avait peu de chance de sortir vivant. D'un autre côté, il se demandait avec inquiétude où l'entraînait la folle surenchère de cette nuit, cette cascade d'inventions sur lesquelles il ne pouvait plus revenir. Si Hodkann parlait, ce serait la catastrophe épouvantable. (p. 102)

Ni le père ni le fils ne parviendront à contenir la puissance active de la rumeur, mais la trahison de Hodkann, qui révélera le secret à la police, est la pire qui puisse advenir. En faisant de Hodkann son complice, Nicolas désobéit à son père, mais il étend, en échange, la communauté du secret et son pouvoir parallèle: il reste dans la logique de la rumeur. En allant à la police, Hodkann livre la rumeur à «l'ennemi», il rompt avec la mystification et le contrepouvoir:

Ce n'est pas le Club des Cinq, Nicolas, ces types sont des monstres [...]: s'il y a une chance de sauver ton père et qu'on la laisse passer, tu ne crois pas que tu te le reprocheras toute ta vie? (p. 124)

Hodkann met un terme au régime discursif de la rumeur et de ses fabulations, il trahit la communauté du secret parce qu'il croit cette histoire véritablement dangereuse. Le meurtre atroce de René confirme à ses yeux la réalité du trafic d'organes, ce qui le décide à trahir Nicolas pour protéger son père.

La catastrophe redoutée par Nicolas arrive donc, mais pas comme prévu. Elle dépasse en horreur tout 
ce qu'il a pu anticiper: en signalant aux policiers que la R 25 grise vue dans le secteur appartient au père de Nicolas, Hodkann livre sans le savoir ce dernier à la justiceet aux médias: le «monstre» dont parlent bientôt les journaux, c'est lui. La rumeur détournée de son circuit parallèle révèle pourtant, dans cet arrêt définitif, le pouvoir magique qui est le sien, pouvoir dangereux qui se retourne contre celui qui l'a transmise à son fils pour le dominer. La conscience du pouvoir magique des fables habite Nicolas. Raconter, pour lui, c'est commettre un crime, comme si la fable pouvait contaminer le réel: «Leur conversation nocturne, ses propres inventions lui faisaient maintenant l'effet d'un crime, d'une participation inavouable, monstrueuse, au crime qui s'était déroulé pour de bon» (p. 107). La rumeur, écrit Françoise Reumaux, est un rite qui fait appel à la "pensée magique» ${ }^{15}$. Dans le cas présent, Nicolas cherche à exorciser la menace par la fabulation, il cherche à éviter le passage à l'acte tout en le redoutant:

Il aurait mieux aimé que Hodkann n'interroge personne [...] et que sous prétexte d'enquête ils se contentent de poursuivre toutes les nuits cette conversation chuchotée, clandestine, rendue excitante par une menace qui n'aurait rien perdu pour lui à rester imaginaire. (p. 118)

Mais l'exorcisme excède le terrain de la fable censée désigner et contenir les démons d'un même élan. Le récit de Nicolas agit sur la réalité: il en modifie d'abord la perception et désigne indirectement, pour finir, le véritable monstre: son père. Les fables se retournent contre celui qui les a diffusées, révélant la vérité cachée de la fascination pour la mort transmise au fils par son père. C'est bien un crime que Nicolas commet alors en le désignant malgré lui comme meurtrier, en révélant involontairement son vrai visage (celui du monstre) aux autorités, aux médias. Mais coupable, il devient aussitôt la victime potentielle de la vengeance collective, le bouc émissaire tout désigné: «[...] si les gens du village apprennent qu'il est ici, dans l'état où ils sont, on ne sait pas de quoi ils sont capables» (p. 138) dit le moniteur. Pour éviter le pire, celui-ci le ramène à son domicile; Nicolas se replie alors dans le silence, seule "protection qu'il pouvait à présent imaginer » (p. 146). Le seuil au-delà duquel la crise éclate est atteint par la médiation de la rumeur qui, en se ruinant, révèle ses pouvoirs au-delà des velléités individuelles de contrôle, de prestige ou d'affranchissement social. La vérité légendaire de la rumeur, celle des monstres dépeceurs d'enfants, est tragiquement confirmée quand Nicolas se voit dépossédé du secret. Le roman se referme devant chez lui, sur le seuil d'une vie désormais prévisible: «Nicolas savait que la porte allait s'ouvrir, qu'à cet instant sa vie commencerait et que dans cette vie, pour lui, il n'y aurait pas de pardon» (p. 148). En faisant basculer la fabulation et les alarmes enfantines dans la tragédie, en resserrant l'intrigue dans une marche «inexorable» vers la catastrophe véritable, le roman ne s'éloigne pas de la rumeur, il en actualise au contraire la profondeur mythique, magique, dramatique et «tragique» ${ }^{16}$. Pour Nicolas, il n'y a plus d'échappatoire possible.

\section{RUMEUR, ROMAN, MODERNITÉ}

La Classe de neige met bien en évidence le lien étroit qui se noue entre rumeur et mythe dans l'espace contemporain. La résonance intérieure que Carrère donne à la rumeur en émergence montre à quel point imaginaires individuel et collectif interagissent au quotidien. Dans les récits circonstanciés, donnés pour véridiques, souvent attestés, couvent des peurs anciennes et des schémas archaïques qui viennent hanter notre modernité. Loin de se situer dans un temps immémorial (in illo tempore) en dehors de l'histoire (voir Eliade, 1969), les mythes que répand la rumeur se manifestent au jour le jour. Ils hantent les imaginaires de la technologie ou de la métropole au gré de l'actualité, surgissent et disparaissent avec elle pour renaitre ensuite, autrement. Dans le circuit qui va de l'information télévisée à la légende des organes volés, Carrère montre en quoi la rencontre confusionnelle entre fiction et réalité n'a rien d'extraordinaire à l'ère des médias de masse (Morin, 1969: 41). L'idée suivant laquelle les moyens d'information actuels peuvent «apaiser une 
inquiétude collective par une sorte de "clinique de la rumeur" "(Delumeau, 2003: 226) ne tient pas compte de la contribution des médias à la confusion entre fiction et réalité, d'où émergent les légendes contemporaines ${ }^{17}$. C'est bien là le milieu favorable à l'éclosion de la rumeur, là où craintes individuelles et collectives se croisent, où information et fantasmes se fondent, où actualité/réalité et archaïsme se mêlent. En ce sens, Nicolas peut être considéré comme la contraction singulière d'un malaise collectif lié à une peur diffuse, omniprésente, qui couve dans toutes les villes du globe, prête à s'actualiser. Dire que cette peur de l'autre ne date pas d'hier est un truisme, rappeler que les "progrès" de l'information participent aujourd'hui de l'émergence de la rumeur est une mise au point nécessaire quand on s'étonne, avec l'affaire d'Outreau par exemple, que l'imaginaire des réseaux malfaisants soit encore aussi virulent au XXI ${ }^{\mathrm{e}}$ siècle ${ }^{18}$.

Le roman de Carrère, que l'on pourrait lire comme un «simple» suspense psychologique, offre l'avantage, d'un point de vue méthodologique, de déplacer la rumeur de son terrain habituel (où elle n'apparaît qu'une fois sa propagation déclarée dans le corps social) pour en isoler l'émergence microscopique et faire du roman lui-même une "caisse de résonance». Plutôt que de ressaisir la circulation de la légende urbaine dans l'espace et les groupes sociaux, il met en relief la mobilité de la rumeur dans les chevauchements de la fiction et du réel au sein d'une intériorité pétrie d'imaginaire collectif que le roman, à son tour, mobilise et réfléchit. Si la rumeur du trafic d'organes ne va pas bien loin dans La Classe de neige, Carrère montre que l'actualisation et la transmission sont nécessaires pour transformer la latence en rumeur active. À suivre l'émergence microscopique de la légende urbaine à travers les tropismes, les images, les informations et les fables qu'elle condense, et en limitant à l'extrême sa propagation, il en répercute les fonctions dramatique et tragique au sein de l'intrigue romanesque, dont le lien "souterrain" avec la rumeur finit par se nouer. En transférant l'imaginaire du réseau «secret» (que raconte la rumeur du trafic d'organes) dans le récit de la circulation confidentielle de la fable et en déplaçant la diffusion en réseau de la rumeur dans le réseau des micro-récits et des motifs, ce sont les affinités "secrètes", "souterraines", entre l'épanouissement de la rumeur et le déploiement romanesque, que Carrère exemplifie et dramatise à son tour, comme par une étrange contamination.

\section{N O TES}

1. Cet article s'inscrit dans le cadre de recherches en cours sur le récit français contemporain subventionnées par le CRSH.

2. Carrère, 1995. Les numéros de page indiqués entre parenthèses après les citations tirées de cet ouvrage renverront désormais à cette édition.

3. Voir Campion-Vincent et Renard, 2002, «Le rein volé», p. 337-345.

4. Les tropismes sont «des mouvements intérieurs ténus, qui glissent rapidement au seuil de notre conscience, $[. .$.$] " ou encore des « actions$ dramatiques intérieures" ("Le Gant retourné», Sarraute, 1996: 1707). "Les tropismes sont des mouvements instinctifs d'expansion et de rétraction " qui passent "par des images très simples et très fortes » qui relèvent souvent d'un imaginaire archaïque où bourreaux et victimes, prédateurs et proies, échangent leurs rôles («Notice», Sarraute, 1996: respectivement 1720 et 1723 ).

5. Je me permets de renvoyer ici à mon article sur L'Adversaire d'Emmanuel Carrère, qui porte en partie sur ce même lieu commun: "L'Avocat du diable? Le Romand d'Emmanuel Carrère", dans Huglo et Rocheville (dir.), 2004: 39-56. Le lieu commun des apparences trompeuses est étroitement lié à la rumeur. Edgar Morin le note à propos de la victime (le bouc émissaire) de la rumeur d'Orléans: «c'est précisément ce qui en fait un être à double-visage: il ressemble à tout le monde et il est autre, il est juif, c'est-à-dire qu'il dissimule sa mystérieuse, son inquiétante différence" (Morin, 1969: 49).

6. Nicolas craint d'abord de s'être vidé de son sang («Il se demanda si son ventre ne s'était pas ouvert, laissant s'écouler ce liquide gluant. Du sang?» (p. 67), puis il se souvient d'une autre histoire épouvantable, La patte de singe, dans laquelle le personnage voyait son corps «se décomposer, se liquéfier, se transformer en un magma noirâtre et visqueux» (p. 68).

7. Carrère, 1995, quatrième de couverture.

8. Le film de Claude Miller (1995) est moins efficace à cet égard que le roman qu'il adapte.

9. Le terme de légende urbaine, employé par Campion-Vincent et Renard (1992) à propos des «rumeurs d'aujourd'hui», indique bien le mélange du légendaire et de l'actuel caractéristique de la rumeur. 10. Rouquette, 1989: 117. Par "problèmes mal définis», Rouquette renvoie à un ensemble de malaises diffus au sein d'un groupe social que la rumeur permet de désigner ponctuellement et, éventuellement, de résorber. 
11. Le fait de "mettre à part» renvoie à l'étymologie du secret: «Le mot français vient de "secretum" en latin, lui-même dérivé de "secretus" et du verbe "secerno", mettre de côté, à l'écart..." (Rabaté, 2000: 18).

12. «Il y a en effet deux langages de la rumeur. Celle-ci se présente soit comme un "on-dit" soit comme un "d'après telle source bien informée” " (Kapferer, 1995: 83).

13. On sait que la rumeur apparaît en «situation de crise [...] telle que le groupe a perdu provisoirement sa sécurité ou son homogénéité " (Rouquette, 1975: 15).

14. Aux rumeurs contemporaines et anciennes mentionnées ici se superpose un autre écho intertextuel, légendaire celui-là, associé à Nicolas: «Ils étaient trois petits enfants / qui s'en allaient glaner aux champs. Tant sont allés tant sont venus / que sur le soir se sont perdus. /S'en sont allés chez le boucher: / "Boucher, voudrais-tu nous loger? / Entrez, entrez, petits enfants, / Y a de la place assurément." / Ils

n'étaient pas sitôt entrés / Que le boucher les a tués, / Les a coupés en p'tits morceaux,/ Mis au saloir comme pourceaux.» («La légende de Saint Nicolas ", Le Livre des chansons de France, Paris, Gallimard, coll. "découverte cadet", 1984, p. 67-68). Dans la chanson, saint Nicolas réveille miraculeusement les trois petits enfants.

15. Il s'agit de "désigner et de décrire une situation " pratique par des objets symboliques et, ce faisant, de la modifier (Reumaux, 1988: 57) 16. Pour F. Reumaux, «la dramatisation et la fonction tragique de la rumeur " la distinguent de «la fonction non tragique mais critique du ragot" (1988: 66).

17. Voir à ce propos les réflexions de M. Augé sur la "fictionnalisation" du présent" (1997: 179).

18. La réouverture du procès d'Outreau en mai 2004 a mis en évidence la difficulté à démêler le vrai du faux dans une enquête judiciaire obsédée par l'idée d'avoir affaire à un réseau de pédophilie. De façon intéressante, les journalistes ont noté la hantise de l'affaire Dutroux dans le procès d'Outreau : l'une a, pour ainsi dire, contaminé l'autre. Voir à ce propos «L'obsession du réseau de pédophiles a conduit aux dérapages de l'enquête» [www.lemonde.fr.], 24 mai 2004.

\section{RÉFÉREN CES BIBLIO GRAPHIQ U ES}

AugÉ, M. [1997]: La Guerre des rêves. Exercices d'ethno-fiction, Paris, Seuil. CAMPION-VinCENT, V. [1988]: «Légendes urbaines. Rumeurs du quotidien, objet d'étude pluridisciplinaire ", Cahiers de littérature orale, $\mathrm{n}^{\circ} 24,75-91$.

CAmpion-Vincent, V. et J. B. Renard [(1992) 2002]: Légendes urbaines. Rumeurs d'aujourd'hui, Paris, Payot \& Rivages.

CARRÈre, E. [1995]: La Classe de neige, Paris, Gallimard, coll. «Folio». Delumeau, J. [(1978) 2003]: La Peur en Occident (XVI-XVIII siècles),

Paris, Hachette littérature.

ELIADE, M. [1969]: Le Mythe de l'éternel retour, Paris, Gallimard, coll.

"Folio-essais".

Huglo, M.P. et S. RocheVILLE (dir.) [2004]: Les Enjeux de la voix narrative dans le récit contemporain, Paris, L'Harmattan, coll.

"Esthétiques".

KAPFERER, J.N. [1995]: Rumeurs. Le plus vieux média du monde, Paris, Seuil, coll. "Points».

MORIN, E. [1969]: La Rumeur d'Orléans. Nouvelle édition complétée avec la rumeur d'Amiens, Paris, Seuil.

RABATÉ, D. [2000]: «Le Secret et la modernité", Modernités, n 14, 9-32. REUMAUX, F. [1988]: «Un rite oral urbain: la rumeur ", Cahiers de littérature orale, $\mathrm{n}^{\circ} 24,55-73$.

ROUQUeTTE, M.L. [1975]: Les Rumeurs, Paris, PUF;

[1989]: «La Rumeur comme résolution d'un problème mal défini ", Cahiers internationaux de sociologie, vol. LXXXVI, janvier-juin, 117122.

SARraute, N. [1996]: CEuvres complètes, Paris, Gallimard, coll.

«Bibliothèque de la Pléiade». 